\title{
CAMA
}

Centre for Applied Macroeconomic Analysis

\section{Non-scale endogenous growth with R\&D and human capital}

\section{CAMA Working Paper 62/2016 October 2016}

\author{
Creina Day
}

Centre for Applied Macroeconomic Analysis, ANU

\begin{abstract}
This paper examines the conditions under which increasing knowledge, encapsulated in ideas for new technology through $R \& D$ and embodied in human capital through education, sustains economic growth. We develop a general model where, consistent with recent literature, growth is non-scale (not increasing in population size) and endogenous (generated by factors within R\&D and education). Recent models feature the counterfactual assumption of constant returns to existing knowledge and restrict the substitutability of inputs within R\&D and education. We find that non-scale endogenous growth is possible under less stringent conditions. Our findings reconcile sustained economic growth with evidence of diminishing marginal returns in education and R\&D, which suggests an ambiguous role for R\&D policy.
\end{abstract}




\section{Keywords}

\section{JEL Classification}

\section{Address for correspondence:}

(E) cama.admin@anu.edu.au

\section{ISSN 2206-0332}

The Centre for Applied Macroeconomic Analysis in the Crawford School of Public Policy has been established to build strong links between professional macroeconomists. It provides a forum for quality macroeconomic research and discussion of policy issues between academia, government and the private sector.

The Crawford School of Public Policy is the Australian National University's public policy school, serving and influencing Australia, Asia and the Pacific through advanced policy research, graduate and executive education, and policy impact. 


\title{
NON-SCALE ENDOGENOUS GROWTH WITH R\&D AND HUMAN CAPITAL
}

\author{
Creina Day \\ CAMA, Australian National University \\ email: creina.day@anu.edu.au
}

\begin{abstract}
This paper examines the conditions under which increasing knowledge, encapsulated in ideas for new technology through REBD and embodied in human capital through education, sustains economic growth. We develop a general model where, consistent with recent literature, growth is non-scale (not increasing in population size) and endogenous (generated by factors within RED and education). Recent models feature the counterfactual assumption of constant returns to existing knowledge and restrict the substitutability of inputs within R\&B and education. We find that non-scale endogenous growth is possible under less stringent conditions. Our findings reconcile sustained economic growth with evidence of diminishing marginal returns in education and $R \mathcal{E} D$, which suggests an ambiguous role for REBD policy.
\end{abstract}

\section{INTRODUCTION}

Increasing knowledge, encapsulated in ideas for new technology and embodied in individuals as human capital, has driven unprecedented growth in living standards for more than a century (Jones and Romer, 2010). A thriving new growth literature (Funke and Strulik, 2000; Dalgaard and Kreiner, 2001; Bucci, 2003; Strulik, 2005; Boonprakaikawe and Tournemaine, 2006; Papageorgiou and Perez-Sebastian, 2006) predicts that research and 
development (R\&D) and human capital accumulation will continue to sustain economic growth under certain conditions. Specifically, recent models draw on constant marginal returns in knowledge accumulation to predict endogenous growth, which means long run growth in the economy is generated within the model rather than by growth in an exogenous factor, in this case, population. ${ }^{1}$ The prediction has relevance for OECD countries facing zero population growth. This paper contributes to the literature by establishing less stringent conditions for endogenous growth, thereby opening up this important field to future research.

To shed light on the origins of conditions for endogenous growth in the recent literature, it is convenient to classify three broad classes of models. First generation models of endogenous growth focus on the accumulation of ideas (Romer, 1990; Aghion and Howitt, 1992) or embodied human capital (Lucas, 1988; Rebelo, 1991) as alternative explanations of long run growth. Constant marginal returns to extant knowledge in the production of new knowledge allow the $\mathrm{R} \& \mathrm{D}$ or education sector to be the engine of growth. Because the marginal product of knowledge does not decline as new knowledge is added over time, R\&D or education can sustain constant growth in technology or human capital, respectively. In this way, the models generate endogenous growth. However, these models predict strong scale effects, whereby an increase in population size permanently raises economic growth ${ }^{2}$, for which there is conflicting evidence. ${ }^{3}$

\footnotetext{
${ }^{1}$ According to the Concise Oxford Dictionary, 'endogenous' means 'having an internal cause or origin'. Growth is endogenous in this sense because the processes of R\&D and human capital accumulation, which are internal to recent models, cause technological change and increasing human capital which sustain long run economic growth.

${ }^{2}$ It is in fact a slight misrepresentation of Romer (1990) and Aghion and Howitt (1992) to say that economic growth is proportional to population. Both models predict that economic growth is proportional to human capital employed in R\&D.

${ }^{3}$ Jones $(1995 a)$ observes a constant growth path in US per capita output over the past century, despite an exponential increase in population. However, Kremer (1993) observes that a region's technological rank reflects its initial population size and there is evidence that economic growth is increasing in the size of the market which reflects initial population size (Sokoloff, 1988, Ciccone and Hall, 1996, Alesina, et al, 2000).
} 
Second generation models focus on the accumulation of ideas through R\&D (Jones, 1995b; Segerstrom 1998; Kortum, 1998) and remove strong scale effects by introducing diminishing marginal returns to extant ideas in $R \& D$. This assumption implies that $R \& D$ requires increasing effort, in the form of a growing population of researchers, to sustain a constant rate of technological change. This is the intuition behind semi-endogenous growth. Jones (1995b) coined the phrase, which means that technological change is endogenous in the sense that it is generated by $R \& D$, but would eventually cease without exogenous population growth. Long run growth is policy invariant and non-scale, meaning it does not increase with population size. ${ }^{4}$

Third generation models consolidate the first generation R\&D-based growth and human capital investment-led models of Romer (1990) and Lucas (1988), respectively, thereby endogenising two types of knowledge, ideas and human capital. These models (Funke and Strulik, 2000; Dalgaard and Kreiner, 2001; Bucci, 2003; Strulik, 2005; Boonprakaikawe and Tournemaine, 2006; Papageorgiou and Perez-Sebastian, 2006) predict non-scale endogenous growth, meaning long run economic growth does not increase with population size and is generated within the model by $R \& D$ and human capital accumulation. ${ }^{5}$ In these models, investment in human capital fuels R\&D, the engine of technological progress that sustains economic growth. The modelling is often highly complex and has developed on an ad hoc basis from the seminal literature on endogenous and semi-endogenous growth.

Recent literature customarily assumes that the elasticity of substitution between factors equals one and that human capital is produced with constant returns. Funke and Strulik (2000), Bucci (2003) and Strulik (2005) assume that R\&D does not draw on existing ideas and features constant returns to the human capital of researchers, which in turn is produced

\footnotetext{
${ }^{4}$ These models exhibit weak scale effects, whereby an increase in population size permanently raises the level of per capita output.

${ }^{5}$ An alternative branch of the literature consolidates the variety and quality ladder R\&D models of Romer (1990) and Aghion and Howitt (1992). The properties of these models are anlaysed by Li (2000).
} 
with constant returns to extant human capital. Boonprakaikawe and Tournemaine (2006) and Papageorgiou and Perez-Sebastian (2006) consider that R\&D draws on extant ideas and features diminishing returns to the human capital of researchers. However, human capital is produced with constant returns to extant human capital. Dalgaard and Kreiner (2001) allow both R\&D and human capital accumulation to draw on existing ideas and human capital. However, they assume that each knowledge sector features constant returns to scale to growing inputs.

There is no apparent reason to expect constant returns in knowledge accumulation. Empirical estimates of $R \& D$ equations do not support the notion that the growth rate of technological knowledge is directly proportional to the scale of resources in $\mathrm{R} \& \mathrm{D}$ (Gong et al, 2004; Ulku, 2007; Bottazzi and Peri, 2007). Trostel (2004) finds evidence of decreasing returns in the production of human capital at the high levels of education attained by countries engaging in R\&D. Furthermore, whether and how R\&D policy may promote long run economic growth depends importantly on marginal returns in R\&D (Funke and Strulik, 2000; Boonprakaikawe and Tournemaine, 2006).

The empirical and policy relevance of non-scale models of endogenous growth warrants careful examination and thorough understanding of their general properties. The purpose of the present paper is twofold. First, this paper analyses a general three sector model which encompasses the recent literature and establishes what conditions are required for equilibrium growth. Second, this paper contributes to the literature by focusing attention on key areas for future research and reconciling non-scale endogenous growth with the lack of empirical support for constant returns to the accumulation of knowledge.

Constant returns are known to be sufficient but not necessary for endogenous growth in one-R\&D-sector and n-R\&D-sector growth models by Christiaans (2004) and Li (2000), respectively. However, no previous attempt that we know of establishes the full set of 
sufficient conditions for non-scale endogenous growth in models with $R \& D$ and human capital accumulation. Previous general models identify conditions for endogenous growth in models with physical capital and human capital accumulation (Rebelo, 1991; Mulligan and Sala-i-Martin, 1993) and conditions for semi-endogenous or endogenous growth in models with physical capital accumulation and R\&D (Eicher and Turnovsky, 1999; Li, 2000; Christiaans, 2004). In doing so, they make an important contribution to our understanding of first and second generation models.

This paper presents a general three sector model of physical capital accumulation, $R \& D$ and human capital accumulation which allows for factors to be productive in all sectors and does not restrict functional forms. Consistent with Jones (2001), population is the only variable that is assumed to accumulate linearly. In the spirit of Uzawa (1961), the analysis focuses on a balanced growth path where variables grow at constant rates. We find the approach of Rebelo (1991), Mulligan and Sala-i-Martin (1993), Eicher and Turnovsky (1999), Li (2000) and Christiaans (2004), which abstracts from microeconomic foundations and models the decision of a central planner, to be a useful starting point for the analysis in this paper which focuses on third generation models of non-scale endogenous growth. Nonetheless, we also show how a decentralised economy can replicate the planning outcome.

\section{General Three-Sector Growth Model}

The model is general in three respects. First, the economy comprises three sectors (final goods, R\&D and human capital accumulation) enabling us to replicate the features of a wide variety of non-scale models of endogenous growth. We model the decision making of a central planner over these sectors. Second, each factor of production is allowed to be productive in each sector. Third, non-parameterised general production functions are employed. Neither parameters nor functional forms are restricted. 
Let $Y_{t}$ denote the output of the final good, $K_{t}$ the stock of physical capital, $A_{t}$ the stock of non-rivalrous knowledge, as measured by the existing variety of ideas for new technology, and $H_{t}$ the stock of human capital at time $t$. Because human capital is embodied, $H_{t}=h L_{t}$, where $h$ is human capital per worker and $L_{t}$ is the stock of labour, which accumulates at the rate of population growth, $n(\dot{L}=n L$ where $n \geq 0)$.

The representative agent of the economy has intertemporal utility of isoelastic form

$$
\int_{0}^{\infty} e^{-\rho t} \frac{c^{1-\theta}}{1-\theta} d t \quad \rho>0 ; \theta>0
$$

where $c_{t}$ denotes consumption per worker at time $t$. Time subscripts are dropped hereafter, for convenience.

Consider the following general three-sector production structure

$$
\begin{aligned}
Y & =F\left(A,\left(1-\alpha_{A}-\alpha_{H}\right) K,\left(1-l_{A}-l_{H}\right) H\right) \\
\dot{A} & =J\left(A, \alpha_{A} K, l_{A} H\right) \\
\dot{H} & =Q\left(A, \alpha_{H} K, l_{H} H\right)
\end{aligned}
$$

where $\alpha_{i}$ and $l_{i}(i=A, H)$ are the fractions of physical capital and labour, respectively, allocated to sector $i$. In the absence of depreciation, physical capital accumulates as a residual after aggregate consumption needs have been met:

$$
\dot{K}=Y-C
$$

where aggregate consumption $C=c L$.

The central planner chooses consumption and the fractions of labour and capital employed in each sector so as to maximise (1) subject to the production and accumulation 
constraints, equations (2a) - (2d). Referring to the appendix, the first order optimality conditions are summarised by

$$
\begin{aligned}
c^{-\theta} & =\lambda L \\
\lambda \frac{F_{H}}{\left(1-l_{A}-l_{H}\right)} & =\mu \frac{J_{H}}{l_{A}}=\gamma \frac{Q_{H}}{l_{H}} \\
\lambda \frac{F_{K}}{\left(1-\alpha_{A}-\alpha_{H}\right)} & =\mu \frac{J_{K}}{\alpha_{A}}=\gamma \frac{Q_{K}}{\alpha_{H}} \\
\rho-\frac{\dot{\lambda}}{\lambda} & =F_{K}+\frac{\mu}{\lambda} J_{K}+\frac{\gamma}{\lambda} Q_{K} \\
\rho-\frac{\dot{\mu}}{\mu} & =\frac{\lambda}{\mu} F_{A}+J_{A}+\frac{\gamma}{\mu} Q_{A} \\
\rho-\frac{\dot{\gamma}}{\gamma} & =\frac{\lambda}{\gamma} F_{H}+\frac{\mu}{\gamma} J_{H}+Q_{H}
\end{aligned}
$$

where $\lambda, \mu$ and $\gamma$ are the shadow values of aggregate physical capital, ideas and human capital, respectively, $F_{i}, J_{i}$ and $Q_{i}$ denote $\partial F / \partial i, \partial J / \partial i$ and $\partial Q / \partial i(i=K, A, H)$. If $n=0$, then $C^{-\theta}=\lambda$ replaces equation (3a). Equations (3b) - (3c) determine the sectoral allocations of labour and capital. ${ }^{6}$ Equations (3d), (3e) and (3f) equate the return to physical capital, ideas and human capital, respectively, to the return on consumption.

A balanced growth path is an equilibrium path where output, consumption, capital, technology and human capital grow at constant, although not necessarily equal, rates (Acemoglu, 2009, page 438). Let $g_{z}$ denote the growth rate in variable $z$, i.e. $g_{z} \equiv \dot{z} / z$. The balanced growth rates of the real variables $(Y, K, A, H)$ are obtained by total differentiation of the production functions (2a) - (2c), noting that constant growth rates requires $g_{Y}=g_{K}{ }^{7}$ $g_{\dot{A}}=g_{A}$ and $g_{\dot{H}}=g_{H}$. A balanced growth equilibrium is therefore characterised by the

\footnotetext{
${ }^{6}$ We solve explicitly for sectoral labour allocations from (3b), (3e) and (3f) and sectoral capital allocations from (3c), (3d) and (3e) in the following section. Pertinent to the following discussion, the sectoral allocations of capital and labour are strictly positive and constant, as required for balanced growth.

${ }^{7}$ The growth rate in physical capital, given by $g_{K}=\frac{\dot{K}}{K}=\frac{Y}{K}-\frac{C}{Y} \frac{Y}{K}$, is constant if $Y, K$ and $C$ grow at the same rate.
} 
following system of three linear equations

$$
\left[\begin{array}{ccc}
\left(1-\sigma_{K}\right) & -\sigma_{A} & -\sigma_{H} \\
-\eta_{K} & \left(1-\eta_{A}\right) & -\eta_{H} \\
-\omega_{K} & -\omega_{A} & \left(1-\omega_{H}\right)
\end{array}\right]\left[\begin{array}{l}
g_{K} \\
g_{A} \\
g_{H}
\end{array}\right]=\left[\begin{array}{l}
0 \\
0 \\
0
\end{array}\right]
$$

where $\sigma_{i} \equiv F_{i} i / F, \eta_{i} \equiv J_{i} i / J$ and $\omega_{i} \equiv Q_{i} i / Q$ denote the structural elasticities in the final production, $R \& D$ and education sectors, respectively, which measure the percentage increase in output of each sector with respect to a percentage increase in each input, $i=K, A, H$. The structural elasticities are not necessarily constant. Intuitively, a structural elasticity captures the marginal return to an input in a sector. For example, $0<\sigma_{K}<1$ captures diminishing marginal returns to $K$ in final production and $\omega_{H}=1$ implies constant marginal returns to $H$ in human capital accumulation.

We assume $\sigma_{i}>0, \eta_{i} \geq 0$ and $\omega_{i} \geq 0 \forall i$, meaning all factors of production are necessary for the production of final output, but some factors of production may not be used in R\&D and human capital accumulation. Without the first assumption, modelling the accumulation of ideas and human capital is inconsequential to the long run growth rate of the economy. Let $A$ denote the coefficient matrix in (4).

Proposition 1: (Necessary condition for strictly positive growth). If growth in output, capital, consumption, ideas and human capital is strictly positive then the coefficient matrix in (4) is singular, $|A|=0$.

This proposition can be shown by the proof in the appendix. Intuitively, singularity of the coefficient matrix means that physical capital accumulation, $R \& D$ and human capital accumulation are just capable of satisfying the growth needs of the three sectors. An important implication is that positive growth in capital, ideas and human capital does not 
require restrictions on specific marginal returns in each sector. While positive growth is provided by Proposition 1, balanced growth requires constancy of the growth rates which solve (4), the conditions for which are summarized in the following proposition.

Proposition 2: $\quad$ (Sufficient conditions for balanced growth). If production functions in all sectors:

1. are Cobb-Douglas (with arbitrary returns to scale satisfying $|A|=0$ ), then balanced growth occurs. In this case, the three sectors may grow at different rates;

2. feature constant returns to scale to endogenously accumulating factors, then balanced growth occurs. In this case, all sectors grow at a common rate, $g_{Y}=g_{K}=g_{A}=g_{H}$.

This proposition can be shown by the proof in the appendix. Intuitively, growth rates in physical capital, ideas and human capital are constant if marginal returns are constant, as for Cobb-Douglas production, or returns to scale are constant, as for Constant Elasticity of Substitution (CES) production. Cobb-Douglas is a special case of CES where the elasticity of substitution between inputs equals one. Thus, this proposition suggests a trade-off between the generality of functional forms and restrictions on the returns to scale.

The solution to (4) implies endogenous growth because positive equilibrium growth does not depend on exogenous population growth. Existing models typically obtain endogenous growth by assuming a linear knowledge accumulation equation. A Lucas (1988) type human capital accumulation equation implies zero entries in the third row of system (4). ${ }^{8}$ However, linear knowledge accumulation is only one of the conditions sufficient for $|A|=0$. The following proposition summarises the full set of conditions that imply endogenous growth.

\footnotetext{
${ }^{8} \mathrm{~A}$ Romer (1990) type R\&D equation where $H$ is an exogenous constant implies zero entries in the second row of a $2 \times 2$ analogue of system (4) where $g_{K}$ and $g_{A}$ are jointly determined.
} 
Proposition 3: $\quad$ (Sufficient conditions for endogenous growth) If either:

1. Human capital is produced with constant marginal returns to existing human capital;

2. All sectors are subject to constant returns to scale to endogenously accumulating factors, in which case all aggregate variables grow at a common rate $g_{Y}=g_{K}=$ $g_{A}=g_{H} ;$ or

3. Increasing (decreasing) returns to scale to endogenously accumulating factors in final production are offset by decreasing (increasing) returns to scale to growing factors in the knowledge accumulation sectors such that $k v_{1}=-\left(v_{2}+v_{3}\right)$, in which case $g_{Y}=g_{K}=k g_{A}=k g_{H}$, where $k \gtrless 1$,

then $|A|=0$.

This proposition can be shown by the proof in the appendix. An important implication of the proposition is that the assumption of linear human capital accumulation which dominates the existing literature is not essential. Similarly, models of endogenous growth where both R\&D and human capital draw on human capital and ideas need not assume constant returns to scale. Intuitively, despite decreasing returns to scale in R\&D and human capital accumulation, increasing returns to scale in final production can generate endogenous growth because doubling physical capital, ideas and human capital more than doubles final output and therefore the accumulation of physical capital, which feeds into R\&D and human capital accumulation.

Proposition 3 differs from earlier work on the conditions for endogenous growth in models of physical and human capital accumulation in one important respect. Each condition in Proposition 3 can be practically applied to models with R\&D and human capital accumulation. Mulligan and Sala-i-Martin (1993) establish that increasing returns to scale 
in one sector, offset by decreasing returns in the other (Interesting Result 4, Example d) is sufficient for endogenous growth. However, Barro and Sala-i-Martin (2004) dismiss an equilibrium in which physical capital to human capital rises or falls forever as implausible. An equilibrium where ideas to human capital rises is plausible, given the non-rivalry of ideas. We herein show how Proposition 3 advances the current literature.

The general model presented in this paper encompasses several recent models of endogenous growth as special cases. We now demonstrate how these recent models can be analysed in the general framework, how their structures fit into our propositions and how our propositions suggest areas for future development.

\section{Linear Human Capital Accumulation and CES Technology}

Recent literature (Funke and Strulik, 2000; Strulik, 2005; Papageorgiou and Perez-Sebastian, 2006) assumes a Lucas (1988) type linear human capital accumulation equation, thereby satisfying Condition 1 of Proposition 3. Consider the following Cobb-Douglas production structure

$$
\begin{aligned}
Y & =A^{\sigma_{A}}\left[\left(1-\alpha_{A}\right) K\right]^{\sigma_{K}}\left[\left(1-l_{A}-l_{H}\right) H\right]^{\sigma_{H}} \\
\dot{A} & =\delta\left[\alpha_{A} K\right]^{\eta_{K}} A^{\eta_{A}}\left(l_{A} H\right)^{\eta_{H}} \\
\dot{H} & =\psi l_{H} H
\end{aligned}
$$

where $\delta$ and $\psi$ are constant efficiency parameters and $\dot{K}=Y-C$.

Along a balanced growth path, positive growth rates in output, ideas and aggregate 
human capital are jointly determined by the system

$$
\left[\begin{array}{ccc}
\left(1-\sigma_{K}\right) & -\sigma_{A} & -\sigma_{H} \\
-\eta_{K} & \left(1-\eta_{A}\right) & -\eta_{H} \\
0 & 0 & 0
\end{array}\right]\left[\begin{array}{l}
g_{K} \\
g_{A} \\
g_{H}
\end{array}\right]=\left[\begin{array}{l}
0 \\
0 \\
0
\end{array}\right]
$$

where $g_{H}=l_{H} \psi$. Recognising $l_{H}$ is constant along a balanced growth path, system (6) reduces to

$$
\left[\begin{array}{cc}
\left(1-\sigma_{K}\right) & -\sigma_{A} \\
-\eta_{K} & \left(1-\eta_{A}\right)
\end{array}\right]\left[\begin{array}{l}
g_{K} \\
g_{A}
\end{array}\right]=\left[\begin{array}{c}
\sigma_{H} l_{H} \psi \\
\eta_{H} l_{H} \psi
\end{array}\right]
$$

with the reduced form solution

$$
\begin{aligned}
g_{Y} & =g_{C}=g_{K}=\frac{\left[\sigma_{H}\left(1-\eta_{A}\right)+\eta_{H} \sigma_{A}\right]}{\left|A_{2}\right|} \psi l_{H} \\
g_{A} & =\frac{\left[\left(1-\sigma_{K}\right) \eta_{H}+\eta_{K} \sigma_{H}\right]}{\left|A_{2}\right|} \psi l_{H}
\end{aligned}
$$

where $\left|A_{2}\right|=\left(1-\sigma_{K}\right)\left(1-\eta_{A}\right)-\eta_{K} \sigma_{A} .^{9}$

Proposition 4: $\quad$ (Necessary and sufficient conditions for positive growth with linear human capital accumulation) $\quad \sigma_{K}<1,\left|A_{2}\right|>0$ (and $\eta_{A}<1$ ) is necessary and sufficient for positive growth of output, capital, consumption, ideas and human capital in a three sector model where $\omega_{H}=1$ and $\omega_{i}=0 \forall i \neq H$.

This proposition can be shown by the proof in the appendix. Intuitively, $\left|A_{2}\right|>0$ means that physical capital accumulation and $R \& D$ are capable of generating more than their individual growth needs, implying the growth rates produced by this group of sectors are positive. Linear technology in the third sector $\left(\omega_{H}=1\right.$ and $\left.\omega_{i}=0 \forall i \neq H\right)$ means

\footnotetext{
${ }^{9}$ With the exception of $\eta_{i}$, all other variables in the above solution are strictly positive by assumption. No further assumptions are required to obtain positive growth of output, although we may reasonably assume that $\eta_{i}>0$ for any but not necessarily all $i \neq A$ to ensure positive growth in ideas.
} 
that human capital accumulation is just capable of satisfying its growth needs. Diminishing returns to physical capital and ideas in their respective sectors $\left(\sigma_{K}<1\right.$ and $\left.\eta_{A}<1\right)$ implies both growth in physical capital and growth in ideas are proportional to growth in human capital.

Recent models share the common assumption that $R \& D$ does not draw on physical capital $\left(\alpha_{A}=\eta_{K}=0\right)$, but differ in the restrictions on other R\&D structural elasticities. Funke and Strulik (2000), Bucci (2003) and Strulik (2005) assume no intertemporal spillovers from ideas $\left(\eta_{A}=0\right)$ and constant marginal returns to research effort in R\&D $\left(\eta_{H}=1\right)$. Papageorgiou and Perez- Sebastian (2006) and Boonprakaikawe and Tournemaine (2006) allow for intertemporal spillovers with diminishing marginal returns from existing ideas $\left(0<\eta_{A}<1\right)$ and diminishing marginal returns from duplication of research effort $\left(0<\eta_{H}<1\right)$. Proposition 4 establishes that the restrictions on $\eta_{i}$ in recent models are more stringent than are needed to obtain positive growth rates in equilibrium. It is not necessary to assume, for example, zero marginal returns to extant ideas or physical capital in $R \& D$.

\section{Example 1: Strict Linearity in Human Capital Accumulation}

It is instructive to show how the innovative phase of Funke and Strulik (2000)'s seminal paper can be analysed as a special case of our general model. The production structure is

$$
\begin{aligned}
& Y=A^{\sigma_{A}} K^{\sigma_{K}}\left[\left(1-l_{A}-l_{H}\right) H\right]^{\sigma_{H}} \quad 0<\sigma_{i}<1 \forall i \\
& \dot{A}=\delta l_{A} H \quad \delta>0 \\
& \dot{H}=\psi l_{H} H \quad \psi>0
\end{aligned}
$$

where $\sigma_{K}=1-\sigma_{H}$ reflects constant returns to scale to private factors, $\dot{K}=Y-C$ and $L=\bar{L}$. Cobb-Douglas production technology satisfies Condition 1 of Proposition 2 . 
The linear human capital accumulation equation (9c) meets Condition 1 of Proposition 3. The condition $\left|A_{2}\right|>0$ in Proposition 4 is met by a stricter condition, $\sigma_{K}=1-\sigma_{H}<1$, which is satisfied since $0<\sigma_{H}<1$.

From (8)

$$
\begin{aligned}
& g_{Y}=g_{C}=g_{K}=\left[\frac{\sigma_{A}}{\sigma_{H}}+1\right] g_{A} \\
& g_{A}=g_{H}=\psi l_{H}
\end{aligned}
$$

where $l_{H}$ is chosen by the central planner. Referring to the appendix, the equilibrium growth rates for the three sectors are

$$
\begin{aligned}
& g_{A}=g_{H}=\frac{(\psi-\rho) \frac{\sigma_{H}}{\sigma_{A}}}{\left[\theta \frac{\sigma_{H}}{\sigma_{A}}+\theta-1\right]} \\
& g_{Y}=\left[\frac{\sigma_{A}}{\sigma_{H}}+1\right] \frac{(\psi-\rho) \frac{\sigma_{H}}{\sigma_{A}}}{\left[\theta \frac{\sigma_{H}}{\sigma_{A}}+\theta-1\right]}
\end{aligned}
$$

where the equilibrium growth rate in per capita income, $g_{y}$, is also given by (11b) since $L=\bar{L}$. Long run economic growth depends neither on growth in an exogenous factor nor on population size and is therefore both endogenous and void of scale effects.

\section{Example 2: CES Human Capital Accumulation}

The linear human capital accumulation equation analysed above suggests that the production of new human capital exactly doubles when the existing stock doubles. However, it seems reasonable to consider that human capital is produced with diminishing marginal returns and that the production of human capital draws on other factors. A CES function allows for varying degrees of substitutability between human capital and other factors. 
As an illustrative example, consider replacing equation (9c) with the CES structure

$$
\dot{H}=\left[\left(\psi_{1} l_{H} H\right)^{\rho}+\left(\psi_{2} \alpha_{H} K\right)^{\rho}\right]^{1 / \rho}
$$

where $\psi_{1}$ and $\psi_{2}$ are constant efficiency parameters, the constant elasticity of substitution between human capital and physical capital is $\epsilon=1 /(1-\rho): \epsilon>0, \epsilon \neq 0$ and the parameters satisfy $\psi_{1} l_{H}>g_{K}>0$. Referring to the appendix, human capital's structural elasticity in the production of human capital is $\omega_{H}=\omega=\left[\left(l_{H} \psi_{1}\right) / g_{H}\right]^{\rho}$. Structural elasticities are variable. However, production technology is constant returns to scale, satisfying Condition 2 of Proposition 1.

Along a balanced growth path

$$
\left[\begin{array}{ccc}
\left(1-\sigma_{K}\right) & -\sigma_{A} & -\sigma_{H} \\
0 & 1 & -1 \\
-(1-\omega) & 0 & (1-\omega)
\end{array}\right]\left[\begin{array}{l}
g_{K} \\
g_{A} \\
g_{H}
\end{array}\right]=\left[\begin{array}{l}
0 \\
0 \\
0
\end{array}\right]
$$

where $\sum \sigma_{i}=1$ is sufficient for $v_{1}=-v_{2}-v_{3}$. Constant returns to growing factors in all sectors satisfies Condition 2 of Proposition 3. In this case, all variables grow at the same rate. Alternatively, if there is more substitutability between human capital and physical capital than for Cobb-Douglas technology, then variables grow at different rates. Referring to the appendix, when $\epsilon>1, g_{H} \rightarrow l_{H} \psi_{1}$ and $\omega \rightarrow 1$. This implies zero entries in the third row of the coefficient matrix. When human capital is highly substitutable for physical capital in production of human capital, equilibrium growth in per capita output asymptotes to

$$
g_{Y} \rightarrow \frac{\left[\sigma_{H}+\sigma_{A}\right]}{\left(1-\sigma_{K}\right)} \bar{g}_{H}=\frac{\left[\sigma_{H}+\sigma_{A}\right]}{\left(1-\sigma_{K}\right)} \psi_{1} l_{H}
$$

where $l_{H}$ is chosen by the central planner. Despite the presence of diminishing marginal 
returns in equation (12), equation (14) compares with equation (10b), because the marginal return of human capital in human capital accumulation tends to a constant when the elasticity of substitution is greater than one.

\section{Examples 1 and 2 with CES production of final goods}

In Examples 1 and 2, the underlying mechanism that generates Cobb-Douglas production is an elasticity of substitution between inputs equal to one, which implies constant structural elasticities. We relax this assumption and replace equation (9a) with the CES function

$$
Y=\left[\left(\beta_{A} A\right)^{a}+\left(\beta_{K} K\right)^{a}+\left(\beta_{H}\left(\left(1-l_{A}-l_{H}\right) H\right)\right)^{a}\right]^{1 / a}
$$

where $\beta_{A}, \beta_{K}$ and $\beta_{H}$ are constant efficiency parameters and the constant elasticity of substitution, which applies to substitution between any two inputs, is $\varepsilon=1 /(1-a)$. Referring to the appendix, the structural elasticities of ideas, human capital and physical capital in overall production are

$$
\begin{aligned}
\sigma_{A} & =\left(\frac{\beta_{A} A}{Y}\right)^{a} \\
\sigma_{H} & =\left(\frac{\beta_{H}\left(l_{Y} H\right)}{Y}\right)^{a} \\
\sigma_{K} & =\left(\frac{\beta_{K} K}{Y}\right)^{a}=1-\left(\sigma_{A}+\sigma_{H}\right)
\end{aligned}
$$

which may vary and sum to one. Constant returns to scale, $\sigma_{A}+\sigma_{H}+\sigma_{K}=1$, satisfies Condition 2 of Proposition 2. Referring to (16a) - (16c), the structural elasticities change as the stocks of $A, H$ and $K$ relative to $Y$ change, which implies a more complex path in transition to balanced growth equilibrium. However, constant returns to scale implies per capita income, consumption and physical capital grow at the same rate in equilibrium. 


\section{Cobb-douglas Technology and Returns to Scale}

While the bulk of the literature assumes linear human capital accumulation, Dalgaard and Kreiner (2001) assume that both existing ideas and human capital are necessary inputs to R\&D and human capital accumulation. This is a special case of Condition 2 of Proposition 3 in two respects. First, production technology is both Cobb-Douglas and constant returns to scale. By Proposition 1, either Cobb-Douglas production technology or constant returns to scale is sufficient for a balanced growth equilibrium. Second, R\&D and human capital accumulation do not draw on physical capital. Condition 2 of Proposition 3 allows for constant returns to scale to all growing factors.

Consider the Cobb-Douglas production structure with both existing ideas and human capital essential to R\&D and human capital accumulation

$$
\begin{array}{ll}
Y=A^{\sigma_{A}}\left(l_{Y} H\right)^{\sigma_{H}} & 0<\sigma_{i}<1 \\
\dot{A}=B_{A} A^{\eta_{A}}\left[l_{A} H\right]^{\eta_{H}} & 0<B_{A}<1 \\
\dot{H}=B_{H} A^{\omega_{A}}\left[l_{H} H\right]^{\omega_{H}} & 0<B_{H}<1
\end{array}
$$

where $H=h L$ and $n>0 . B_{A}$ and $B_{H}$ are exogenous shift parameters which measure the share of total output invested in R\&D and human capital accumulation, respectively, in the special case of identical production technology,

From (17), the long run growth rate of the economy is given by $g_{y}=\sigma_{A} g_{A}+\sigma_{H} g_{H}-n$, which is constant, as required for balanced growth, if both $g_{A}$ and $g_{H}$ are constant. In a balanced growth equilibrium, positive growth rates in ideas and aggregate human capital 
are jointly determined by the system

$$
\left[\begin{array}{cc}
\left(1-\eta_{A}\right) & -\eta_{H} \\
-\omega_{A} & \left(1-\omega_{H}\right)
\end{array}\right]\left[\begin{array}{l}
g_{A} \\
g_{H}
\end{array}\right]=\left[\begin{array}{l}
0 \\
0
\end{array}\right]
$$

where $|A|=0$ is necessary for a solution and $v_{2}=-k v_{1}$ is sufficient for $|A|=0$. Along a balanced growth path, the growth rate of ideas satisfies

$$
g_{A}=\left[\frac{\eta_{H}+\left(1-\omega_{H}\right)}{\left(1-\eta_{A}\right)+\omega_{A}}\right] g_{H}=k g_{H}
$$

where $k<(>) 1$ denotes decreasing (increasing) returns to scale in $\mathrm{R} \& \mathrm{D}$, respectively, with offsetting increasing (decreasing) returns to scale in human capital accumulation and $g_{A}<(>) g_{H}$ if and only if $k<(>) 1$.

To shed light on the prevalence of constant returns to scale in the literature, we consider the lab equipment framework (Rivera-Batiz and Romer, 1991, Acemoglu, 2009, Chapter 13.1), which assumes production technology is identical across sectors.

Proposition 5: In a two knowledge sector Cobb-Douglas model where sectors share identical production technology, constant returns to scale is necessary and sufficient for balanced growth.

This proposition can be shown by the proof in the appendix. Intuitively, if the marginal returns to ideas and human capital are identical across $R \& D$ and human capital accumulation then the evolution of growth in ideas determines growth in human capital, and thus output which draws only on ideas and human capital. Constant growth in ideas requires constant returns to scale, which implies that $R \& D$ is just capable of generating its growth needs. Increasing or decreasing returns to scale would imply explosive of ever-decreasing growth, respectively. 


\section{Example 3: Identical technology and constant returns to scale}

Dalgaard and Kreiner (2001) apply the lab equipment framework, whereby ideas and human capital accumulate from shares of final production. Consider the special case of (17) where $\sigma_{H}=\eta_{H}=\omega_{H}, \sigma_{A}=\eta_{A}=\omega_{A}$ and $l_{Y}=l_{A}=l_{H}=1$, such that $\dot{A} / Y=B_{A}$ and $\dot{H} / Y=B_{H}$. Along a balanced growth path

$$
\left[\begin{array}{cc}
\left(1-\sigma_{A}\right) & -\sigma_{H} \\
-\sigma_{A} & \left(1-\sigma_{H}\right)
\end{array}\right]\left[\begin{array}{l}
g_{A} \\
g_{H}
\end{array}\right]=\left[\begin{array}{l}
0 \\
0
\end{array}\right]
$$

where $v_{1}=-v_{2}$, sufficient for $|A|=0$, is satisfied if $\sigma_{A}+\sigma_{H}=1$. Consistent with Proposition 5, constant returns to scale becomes a stricter condition for balanced growth because R\&D and human capital accumulation share identical production technology.

System (20) provides one equation with two unknowns which implies $g_{A}=g_{H}$. Referring to the appendix, the equilibrium growth rates for ideas, human capital and per capita output are

$$
\begin{aligned}
& g_{A}=g_{H}=g_{h}+n=\left(B_{A}\right)^{1-\sigma_{H}}\left(B_{H}\right)^{\sigma_{H}} \\
& g_{y}=\left(B_{A}\right)^{1-\sigma_{H}}\left(B_{H}\right)^{\sigma_{H}}-n
\end{aligned}
$$

where the optimal shares of output are chosen by a central planner. Long run economic growth, $g_{y}$, depends negatively on population growth. By implication, the long run level of per capita output, $y$, is also void of scale effects. Intuitively, constant returns to scale implies ideas and aggregate human capital grow at a common rate in equilibrium, but population growth reduces human capital growth of the average individual in the population due to a capital dilution effect.

It is useful to consider the plausibility of the different examples. If physical capital's 
share of output is $\hat{\sigma}_{K}=0.36$ (Jones and Williams, 2000, Papageorgiou and Perez-Sebastian, 2006), the assumption $\sigma_{K}+\sigma_{H}=1$ in Examples 1 and 2 implies $\hat{\sigma}_{H}=0.64$, which is consistent with recent evidence that $\hat{\sigma}_{H}=0.6$ is a lower bound (de la Fuente, 2011). For $\hat{\sigma}_{A}=0.1$ (Papageorgiou and Perez-Sebastian, 2006), final production exhibits modest increasing returns to scale. Evidence of increasing returns to scale in final production supports Example 1. However, referring to $(10), \hat{\sigma}_{A}=0.1$ and $\hat{\sigma}_{H}=0.64$ imply $g_{K}=$ $1.15 g_{H}$, which means $K / H$ rises forever in equilibrium.

Trostel (2004) tests the relationship between logarithm wages and years of education for marginal returns in human capital accumulation and estimates a marginal rate of return to education of around 0.1. Thus, Examples 2 and 3 which allow for $0<\omega_{H}<1$ in human capital accumulation may be more plausible than Example 1 which requires constant returns to human capital.

Gong et al (2004) estimate $\hat{\eta}_{H}=0.5$ when they impose the restriction $\eta_{A}=1$, and $\hat{\eta}_{A}=0.1$ without the restriction. Jones and Williams (2000) find that $\hat{\eta}_{H}=0.5$ is a lower bound when $\eta_{A}$ is unrestricted. Thus, Example 3 which assumes $0<\eta_{H}<1$ and $0<\eta_{A}<1$ may be more plausible. This assumption has interesting implications for the role of the central planner with respect to policy, which we assess in the following section.

\section{The DeCEntralised ECONOMY}

The growth rates for a corresponding decentralised economy differ only by the absence of terms, such as a monopoly markup, that capture the inefficiencies that a planner internalises. Inefficiencies can arise in $R \& D$ and the use of goods embodying ideas in final production (Acemoglu, 2009, Chapter 13.1). We herein show that it is possible for the decentralised economy to replicate the planning outcome.

A decentralised economy comprises a representative household and firms in the final 
goods, intermediate goods and $\mathrm{R} \& \mathrm{D}$ sectors. The $\mathrm{R} \& \mathrm{D}$ sector produces ideas encapsulated in designs for new intermediate goods. Firms in the intermediate goods sector own the patents for designs, enabling manufacture and sale of intermediate goods to the final goods sector at a price above marginal cost. The framework is one of monopolistic competition, where monopoly profits are extracted by the R\&D sector in the form of compensation for research effort.

An index of intermediate goods is represented by

$$
\left[\int_{0}^{A} x(j)^{\phi} d j\right]^{1 / \phi} \quad 0<\phi<1
$$

where $A$ denotes the number of intermediate goods, $x(j)$ is the input of intermediate good $j$ and $\phi$ determines a constant price elasticity of demand for intermediates, $1 /(1-\phi)>1$. Since the intermediate goods sector is symmetric, the quantity $x(j)=x$ of each intermediate good is employed, simplifying equation (22) to $A^{1 / \phi} x$. Thus,

$$
Y=F\left(A^{1 / \phi} x,\left(1-\alpha_{A}-\alpha_{H}\right) K,\left(1-l_{A}-l_{H}\right) H\right)
$$

and

$$
\dot{K}=Y-C-A x
$$

since consumption, $C$, investment, $\dot{K}$, and intermediate goods are all produced with the same technology as final production.

It is instructive to derive in detail the decentralised outcome for Example 1. Consider

$$
Y=\left[A^{1 / \phi} x\right]^{\sigma_{A}} K^{\sigma_{K}}\left[\left(1-l_{A}-l_{H}\right) H\right]^{\sigma_{H}} \quad 0<\sigma_{i}<1 \forall i
$$

where $\sigma_{K}=1-\sigma_{H}$ and $L=\bar{L}$. Referring to the appendix, the decentralised economy 
allocates

$$
l_{Y}^{D}=\frac{1}{1-\phi} \frac{\psi}{\delta} \frac{\sigma_{H}}{\sigma_{A}} \frac{A}{H}
$$

to final production. The equilibrium growth rates are

$$
\begin{aligned}
& g_{A}=g_{H}=\frac{(\psi-\rho) \frac{\phi}{1-\phi} \frac{\sigma_{H}}{\sigma_{A}}}{\frac{\phi}{1-\phi} \frac{\sigma_{H}}{\sigma_{A}} \theta+\theta-1} \\
& g_{Y}=\left[\frac{1-\phi}{\phi} \frac{\sigma_{A}}{\sigma_{H}}+1\right] \frac{(\psi-\rho) \frac{\phi}{1-\phi} \frac{\sigma_{H}}{\sigma_{A}}}{\frac{\phi}{1-\phi} \frac{\sigma_{H}}{\sigma_{A}} \theta+\theta-1}
\end{aligned}
$$

where market structure affects growth via parameter $\phi$.

Consider that a benevolent planner chooses consumption, intermediate goods and the fractions of labour and capital employed so as to maximise (1) subject to (23), (2b), (2c) and (24). The first order optimality conditions are (3a) - (3f) where

$$
\rho-\frac{\dot{\mu}}{\mu}=\frac{\lambda}{\mu}\left(F_{A}-x\right)+J_{A}+\frac{\gamma}{\mu} Q_{A}
$$

replaces (3e) and

$$
\lambda\left[F_{A}-x\right]=0
$$

gives the optimal choice of $x$. Referring to the appendix, the planner allocates

$$
l_{Y}^{C P}=\frac{\phi}{1-\phi} \frac{\psi}{\delta} \frac{\sigma_{H}}{\sigma_{A}} \frac{A}{H}
$$

which is $(1-\phi)$ less than the equilibrium share of labour allocated to final production in a decentralised economy. A subsidy to intermediate goods demand of $(1-\phi)$ would address insufficient allocation of labour to knowledge accumulation in the decentralised economy. However, the equilibrium growth rates in ideas, human capital and per capita income are the same as (27a) and (27b). Intuitively, a benevolent planner allocates a higher 
share of labour to R\&D and human capital accumulation, which increases the level of per capita output, but it is the rate of growth in ideas and human capital that drives long run economic growth (Funke and Strulik, 2000).

This example assumes that no intertemporal spillovers $\left(\eta_{A}=0\right)$ and constant marginal returns to human capital $\left(\eta_{H}=1\right)$ in $\mathrm{R} \& \mathrm{D}$. Our general model allows for less stringent parameter assumptions which imply distortions in the R\&D sector. First, intertemporal spillovers $\left(\eta_{A}>0\right)$ suggest that researchers underinvest because they cannot appropriate the value of spillovers to future $R \& D$. Second, diminishing marginal returns to human capital $\left(0<\eta_{H}<1\right)$ suggest that researchers overinvest because they duplicate the effort of other researchers.

Equilibrium growth in a decentralised economy may be greater or less than the social optimum, depending on the relative effect of positive intertemporal spillovers and negative duplication externalities in $R \& D$. Thus, whether $R \& D$ is subsidised or taxed to implement the optimum is ambiguous. This finding is consistent with the policy analysis in specific models with R\&D intertemporal spillovers and diminishing marginal returns to human capital (Jones and Williams, 2000, Boonprakaikawe and Tournemaine, 2006).

\section{CONCLUSION}

Non-scale models of endogenous growth assume that knowledge is produced with CobbDouglas technology and constant returns to extant knowledge. We establish that endogenous growth without scale effects is feasible under a broader set of conditions than feature in the existing literature. First, either Cobb-Douglas or CES production imply balanced growth. Second, human capital produced with either (i) constant marginal returns to extant human capital; (ii) constant returns to scale to human capital and other growing factors; or (iii) decreasing returns to scale, offset by increasing returns to scale in R\&D, is 
sufficient for balanced growth. Constant returns are necessary and sufficient for balanced growth only if production is CES or ideas and human capital accumulate from identical production technology. In general, returns to scale may vary, which in turn implies that output, ideas and human capital grow at different rates. Finally, in the case of linear human capital accumulation, diminishing marginal returns to physical capital in final production and existing ideas in R\&D is necessary and sufficient for long run economic growth.

The results in this paper help frame the research agenda going forward. The first result suggests that long run economic growth can be sustained if production is CES. It seems reasonable to expect that the elasticity of substitution between factors is not exactly and always equal to one, especially as the number of sectors in a model of endogenous growth increase. The second result reconciles the use of Cobb-Douglas technology with increasing returns to scale in R\&D (Romer, 1990) and evidence of diminishing returns in human capital accumulation. An equilibrium in which the ratio of ideas to human capital rises may be plausible, given the non-rivalry of ideas and is empirically consistent. The final result reduces the number of necessary parameter restrictions in final production and R\&D for the recent literature which customarily assumes linear human capital accumulation.

There are some natural connections with the Uzawa (1961) theorem which only exploits the fact that the production function exhibits constant returns to scale and that output, capital and consumption grow at a constant rate in balanced growth. Similarly, the results on balanced growth in this paper are established without reference to the detailed equilibrium behaviour of endogenous growth models with $R \& D$ and human capital. It is, nonetheless, possible for a model of a decentralised economy to replicate the balanced growth equilibrium. Interestingly, the empirically supported assumption of diminishing marginal returns to ideas and human capital in R\&D suggests an ambiguous role for policy. 


\section{REFERENCES}

Acemoglu, D. (2009). Introduction to modern economic growth, Princeton University Press, Princeton, NJ.

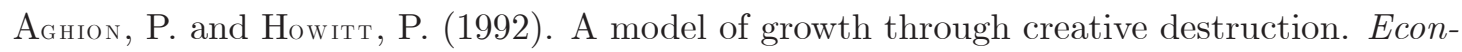
ometrica, 60, pp. 323-512.

Alesina, A. Spolaore, E. and Wacziarg, R. (2000). Economic integration and political disintegration. American Economic Review, 90, pp. 12761296.

Barro, R.J. and Salai-i-martin, X. (2004). Economic Growth, Second edition, MiT Press, Cambridge, MA.

Boonprakaikane, J. and Tournemaine, F. (2006). Production and consumption of education in a R\&D-based growth model. Scottish Journal of Political Economy, 53, pp. 565-585.

Bотtazzi, L. and Peri, G. (2007). The dynamics of R\&D and innovation in the short run and in the long run. The Economic Journal, 117, pp. 486-511.

BuccI, A. (2003). R\&D, imperfect competition and growth with human capital accumulation. Scottish Journal of Political Economy, 50, pp. 417-439.

Christianan, T. (2004). Types of balanced growth. Economics Letters, 82, pp. 253-58.

Ciccone, A. and Hall, R. (1996). Productivity and the density of economic activity. American Economic Review, 86, pp. 54-70.

Dalgaard, C. and Kreiner, C. (2001). Is declining productivity inevitable? Journal of Economic Growth, 6, pp. 187-203.

De la fuente, A. (2011). Human capital and productivity. Nordic Economic Policy Review, 2, pp. 103-132.

Eicher, T. and Turnovsky, S. (1999). Non-scale models of economic growth. The Economic Journal, 109, pp. 394-415.

Funke, M. and Strulik, H. (2000). On endogenous growth with physical capital, human 
capital and product variety. European Economic Review, 44, pp. 491-515.

Gong, G., Greiner, A. and Semmler, W. (2004). Endogenous growth: Estimating the Romer model for the United States and Germany. Oxford Bulletin of Economics and Statistics, 66, 147-64.

Hawrins, D. and Simon, H. A. (1949). Note: Some conditions of macroeconomic stability. Econometrica, 17, pp. 245-48.

Jones, C.I. (1995a). Time series tests of endogenous growth models. Quarterly Journal of Economics, 110, pp. 494-525.

Jones, C.I. (1995b). R\&D-based models of economic growth. Journal of Political Economy, 103, pp. 759-83.

Jones, C.I. (2001). Population and ideas: a theory of endogenous growth. Working Paper, University of California, Berkeley. Version 5.0.

Jones, C.I. and Romer, P.M. (2010). The new Kaldor facts: ideas, institutions, population and human capital. American Economic Journal: Macroeconomics, 2, pp. 224-45.

Jones, C.I. and Williams, J.C. (2000). Too much of a good thing? The economics of investment in R\&D. Journal of Economic Growth, 5, pp.65-85.

Kremer, M. (1993). Population growth and technological change: One million B.C. to 1990. Quarterly Journal of Economics, 108, pp. 681-716.

Kortum, S.S. (1997). Research, patenting and technological change. Econometrica, 65, pp. 1389-1419.

L. C.W. (2000). Endogenous versus semi-endogenouos growth in a two-R\&D-sector model. The Economic Journal, 110, C109-C122.

Lucas, R. (1988). On the mechanics of economic development. Journal of Monetary Economics, 22, pp. 3-41.

Muldigan, C.B. and Sala-i-martin, X. (1993). Transitional dynamics in two-sector models 
of endogenous growth. Quarterly Journal of Economics, 108, pp. 739-75.

Papageorgiou, C. and Perez-sebastian, F. (2006). Dynamics in a non-scale R\&D growth model with human capital: Explaining the Japanese and South Korean development experiences. Journal of Economic Dynamics and Control, 30, pp. 901-30.

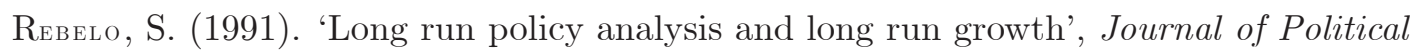
Economy, vol. 99, pp. 5-21.

Rivera-Batiz, L. A. and Romer, P. M. (1991). Economic integration and endogenous growth. Quarterly Journal of Economics, 106, pp. 531-55.

Romer, P.M. (1990). Endogenous technological change. Journal of Political Economy, 98, S71-S102.

Segerstrom, P. (1998). Endogenous growth without scale effects. American Economic Review, 88, pp. 1290-310.

Sокоцоғғ, K. (1988). Inventive activity in early industrial America: Evidence from patent records, 1790-1846. The Journal of Economic History, 48, pp. 813-850.

Strulik, H. (2005). The role of human capital and population growth in R\&D-based models of economic growth. Review of International Economics, 13, pp. 129-45.

Sydsaeter, K., Hammond, P. and Strom, A. (2012) Essential Mathematics for Economic Analysis, fourth edition, Pearson.

Trostel, P. (2004). Returns to scale in producing human capital from schooling. Oxford Economic Papers'. 56, pp. 461-84.

$\mathrm{ULKU}_{\mathrm{LK}}$, H. (2007). R\&D, innovation, and growth: evidence from four manufacturing sectors in OECD countries. Oxford Economic Papers, 59, pp. 513-35.

Uzawa, H. (1961). Neutral inventions and the stability of growth equilibrium. Review of Economic Studies, 28, pp. 117-24. 


\section{APPENDIX}

Derivation of first order optimality conditions

The current value Hamiltonian is

$$
\hat{H}=\frac{c^{1-\theta}}{1-\theta}+\lambda[F(.)-c L]+\mu J(.)+\gamma Q(.)
$$

where the control variables of this problem are $c, l_{A}, l_{H}, \alpha_{A}, \alpha_{H}$ and the state variables are $K, A, H$. The first order optimality conditions are

$$
\begin{aligned}
\hat{H}_{c} & =c^{-\theta}-\lambda L=0 \\
\hat{H}_{l_{A}} & =-\lambda F_{l_{Y} H} H+\mu J_{l_{A} H} H=0 \\
\hat{H}_{l_{H}} & =-\lambda F_{l_{Y} H} H+\gamma Q_{l_{H} H} H=0 \\
\hat{H}_{\alpha_{A}} & =-\lambda F_{\alpha_{Y} K} K+\mu J_{\alpha_{A} K} K=0 \\
\hat{H}_{\alpha_{H}} & =-\lambda F_{\alpha_{Y} K} K+\gamma Q_{\alpha_{H} K} K=0 \\
\dot{\lambda}-\rho \lambda & =-\hat{H}_{K}=-\lambda F_{K}-\mu J_{K}-\gamma Q_{K} \\
\dot{\mu}-\rho \mu & =-\hat{H}_{A}=-\lambda F_{A}-\mu J_{A}-\gamma Q_{A} \\
\dot{\gamma}-\rho \gamma & =-\hat{H}_{H}=-\lambda F_{H}-\mu J_{H}-\gamma Q_{H}
\end{aligned}
$$

where $\hat{H}_{j}$ denotes $\partial \hat{H} / \partial j\left(j=c, l_{A}, l_{H}, \alpha_{A}, \alpha_{H}, K, A, H\right)$ and $F_{j}, J_{j}, Q_{j} \operatorname{denotes} \partial F / \partial j, \partial J / \partial j, \partial Q / \partial j$, respectively, plus the transversality conditions

$$
\lim _{t \rightarrow \infty} e^{-\rho t} \lambda K=\lim _{t \rightarrow \infty} e^{-\rho t} \mu A=\lim _{t \rightarrow \infty} e^{-\rho t} \gamma H=0
$$

Equating (FOC2) and (FOC3), substituting for $F_{l_{Y} H}=F_{H} / l_{Y}, J_{l_{A} H}=J_{H} / l_{A}, Q_{l_{H} H}=$ $Q_{H} / l_{H}$, gives (3b). Equating (FOC4) and (FOC5), substituting for $F_{\alpha_{Y} K}=F_{K} / \alpha_{Y}, J_{\alpha_{A} K}=$ 
$J_{K} / \alpha_{A}, Q_{K} K / \alpha_{H}$,gives (3c).

\section{Proof of Proposition 1}

The system of linear equations in (4) is homogeneous (in matrix form $A x=0$ ). A homogeneous linear system of equations has non-trivial solutions if and only if the coefficient matrix is singular, i.e. $|A|=0$ (Theorem 13.7, Sydsaeter et al, 2012). Thus, (4) has a solution other than $g_{K}=g_{A}=g_{H}=0$ iff $|A|=0$. The existence of a solution with positive growth rates implies $|A|=0$. However, $|A|=0$ does not imply positive growth since a non trivial solution may be one of negative growth. Thus, $g_{K}>0, g_{A}>0, g_{H}>0 \Rightarrow|A|=0$.

\section{Proof of Proposition 2}

From (4),

$$
g_{Y}=g_{K}=x_{1} g_{A}+x_{2} g_{H}
$$

where $x_{1}=\left[\frac{\sigma_{A}+\left(1-\eta_{A}\right)-\omega_{A}}{\left(1-\sigma_{K}\right)+\eta_{K}+\omega_{K}}\right]$ and $x_{2}=\left[\frac{\sigma_{H}-\eta_{H}+\left(1-\omega_{H}\right)}{\left(1-\sigma_{K}\right)+\eta_{K}+\omega_{K}}\right]$. Letting $\bar{x}$ denote constant $x$,

$$
\bar{x}_{1}, \bar{x}_{2} \Rightarrow \bar{g}_{Y}=\bar{g}_{K}, \bar{g}_{A}, \bar{g}_{H}
$$

where

$$
\begin{aligned}
\bar{\sigma}_{i}, \bar{\eta}_{i}, \bar{\omega}_{i} & \Rightarrow \bar{g}_{Y}=\bar{g}_{K}=\bar{x}_{1} \bar{g}_{A}+\bar{x}_{2} \bar{g}_{H} \\
\sum \sigma_{i} & =1, \sum \eta_{i}=1, \sum \omega_{i}=1 \Rightarrow \bar{g}_{Y}=\bar{g}_{K}=\bar{g}_{A}=\bar{g}_{H}
\end{aligned}
$$

\section{Proof of Proposition 3}

Let $v_{i}$ denote the $i^{\text {th }}$ column vector of the coefficient matrix $A$.

$$
v_{1}=a v_{2}+b v_{3} \quad(a<0 ; b<0) \quad \Rightarrow|A|=0
$$


Each of the following cases implies $|A|=0$, as required for a solution to system (4):

1. Zero entries in the third row is provided by $\omega_{\cdot H}=1 ; \omega_{i}=0 \forall i \neq H \Rightarrow|A|=0$.

2. $\sum \sigma_{i}=1 ; \sum \eta_{i}=1 ; \sum \omega_{i}=1 \forall i \Rightarrow v_{1}=-v_{2}-v_{3}$. Set $g_{A}=g_{H}$ in (P2). Substituting $\sum \sigma_{i}=1 ; \sum \eta_{i}=1 ; \sum \omega_{i}=1 \forall i$ gives $g_{Y}=g_{K}=g_{A}=g_{H}$.

3. Increasing (decreasing) returns to scale to endogenously accumulating factors in final production correspond to $\sum \sigma_{i} \gtrless 1$, respectively. Let $\left(1-\sigma_{K}\right)=\sigma>0$. The relationship $k v_{1}=-\left(v_{2}+v_{3}\right) \Rightarrow \sum \sigma_{i}=1+(k-1) \sigma \gtrless 1 \Leftrightarrow k \gtrless 1$. Similarly, $\sum \eta_{i} \lessgtr 1 \Leftrightarrow k \gtrless 1$ and $\sum \omega_{i} \lessgtr 1 \Leftrightarrow k \gtrless 1$. Substituting $\left(\sigma_{A}+\sigma_{H}\right)=k\left(1-\sigma_{K}\right)$, $\left(\eta_{A}+\eta_{H}\right)=\left(1-k \eta_{K}\right)$ and $\left(\omega_{A}+\omega_{H}\right)=\left(1-k \omega_{K}\right)$ gives $g_{Y}=g_{K}=k g_{A}=k g_{H}$.

\section{Proof of Proposition 4}

The system of equations in (7) is non-homogenous, $A x=d$ in matrix notation. By Hawkins and Simon's (1949) theorem, a necessary and sufficient condition that $g_{K}$ and $g_{A}$ satisfying (7) be positive is that all principal minors of the coefficient matrix $A$ be positive. Denoting $\left|A_{i}\right|$ as the $i^{\text {th }}$ principal minor of the coefficient matrix, $\left|A_{1}\right|=\left(1-\sigma_{K}\right)>0$ and $\left|A_{2}\right|=$ $\left(1-\sigma_{K}\right)\left(1-\eta_{A}\right)-\eta_{K} \sigma_{A}>0$ imply restrictions on the structural elasticities. $\left|A_{1}\right|>0 \Leftrightarrow$ $\sigma_{K}<1$, while $\left|A_{1}\right|>0$ and $\left|A_{2}\right|>0$ together imply $\eta_{A}<1$.

\section{Proof of Proposition 5}

If $\eta_{A}=\omega_{A}$ and $\eta_{H}=\omega_{H}$, then $g_{A}=g_{H}$. The dynamics of $g_{Y}$ is determined by the dynamics of $g_{A}$,

$$
\dot{g}_{A}=\left(\eta_{A}-1\right) g_{A}+\eta_{H} g_{H}=\left(\eta_{A}+\eta_{H}-1\right) g_{A}
$$

where $\dot{g}_{A} \gtreqless 0 \Leftrightarrow\left(\eta_{A}+\eta_{H}-1\right) \gtreqless 0 . \quad \dot{g}_{A} \gtrless 0$ is inconsistent with balanced growth. Therefore, $\eta_{A}+\eta_{H}=1 \Leftrightarrow \dot{g}_{A}=\dot{g}_{H}=0$. 


\section{Derivation of Equilibrium Growth Rates}

Example 1: Linear Human Capital Accumulation From (8)

$$
\begin{aligned}
& g_{Y}=g_{C}=g_{K}=\left[\frac{\sigma_{A}}{\sigma_{H}}+1\right] g_{A} \\
& g_{A}=g_{H}
\end{aligned}
$$

Differentiating (3a) with respect to time, substituting from (3d) for $g_{\lambda}$ and $F_{K}=r$,

$$
g_{C}=\frac{1}{\theta}(r-\rho)
$$

From the second equality in (3b), $\mu \delta=\gamma \psi$. Differentiating with respect to time, we can substitute for $\dot{\mu}$ and $\mu$ in (3e). Equating with (3f) and further substituting for $\mu \delta$ and $\gamma \psi$ in $(3 \mathrm{f})$

$$
l_{Y}=\frac{\psi}{\delta} \frac{\sigma_{H}}{\sigma_{A}} \frac{A}{H}
$$

which is constant, since $A / H$ is the steady state variable from (A1.2).

From (3d)

$$
\frac{\dot{\lambda}}{\lambda}=\rho-r
$$

Substituting from (3b) and $1=l_{Y}+l_{A}+l_{H}$ in (3f)

$$
\frac{\dot{\gamma}}{\gamma}=\rho-\psi
$$

Differentiating (3b) with respect to time and substituting for (A1.5) and (A1.6)

$$
g_{Y}-g_{l_{Y}}-g_{H}=r-\psi
$$


where substituting for $g_{l_{Y}}=0, r$ and $g_{Y}$ from (A1.4), (A1.3) and (A1.1), respectively, gives

$$
g_{A}=g_{H}=\frac{(\psi-\rho) \frac{\sigma_{H}}{\sigma_{A}}}{\left[\theta \frac{\sigma_{H}}{\sigma_{A}}+\theta-1\right]}
$$

Example 2: CES Human Capital Accumulation From $\omega_{H} \equiv \frac{Q_{H} H}{Q}$ and $\omega_{K} \equiv \frac{Q_{K} K}{Q}$,

$$
\begin{aligned}
\omega_{H} & =\frac{\left(\psi_{1} l_{H} H\right)^{\rho}}{\left[\left(\psi_{1} l_{H} H\right)^{\rho}+\left(\psi_{2} \alpha_{H} K\right)^{\rho}\right]} \\
& =\left(\frac{\left(\psi_{1} l_{H} H\right)}{\left[\left(\psi_{1} l_{H} H\right)^{\rho}+\left(\psi_{2} \alpha_{H} K\right)^{\rho}\right]^{1 / \rho}}\right)^{\rho} \\
& =\left(\frac{\psi_{1} l_{H}}{g_{H}}\right)^{\rho} \equiv \omega \\
\omega_{K} & =\frac{\left(\psi_{2} \alpha_{H} K\right)^{\rho}}{\left[\left(\psi_{1} l_{H} H\right)^{\rho}+\left(\psi_{2} \alpha_{H} K\right)^{\rho}\right]} \\
& =\frac{\left(\psi_{1} l_{H} H\right)^{\rho}+\left(\psi_{2} \alpha_{H} K\right)^{\rho}-\left(\psi_{1} l_{H} H\right)^{\rho}}{\left[\left(\psi_{1} l_{H} H\right)^{\rho}+\left(\psi_{2} \alpha_{H} K\right)^{\rho}\right]} \\
& =1-\left(\frac{\psi_{1} l_{H}}{g_{H}}\right)^{\rho}=1-\omega
\end{aligned}
$$

Differentiating $g_{H}=\frac{\dot{H}}{H}=\frac{Q\left(l_{H} H, \alpha_{H} K\right)}{H}$ with respect to time and noting that $i_{H}=0$ and $\dot{\alpha}_{H}=0$ along a balanced growth path, and $\frac{Q_{H} H}{Q}=\omega$ and $\frac{Q_{K} K}{Q}=1-\omega$, yields

$$
\dot{g}_{H}=g_{H}\left(g_{K}-g_{H}\right)\left\{1-\left(\frac{\psi_{1} l_{H}}{g_{H}}\right)^{\rho}\right\}
$$

which implies a stable steady state of $g_{K}$ or $l_{H} \psi_{1}$, depending on whether $\epsilon<1$ or $\epsilon>1$, 
respectively.

$C E S$ Final Production From $\sigma_{A} \equiv \frac{F_{A} F}{F}, \sigma_{K} \equiv \frac{F_{K} F}{F}$ and $\sigma_{H} \equiv \frac{F_{H} F}{F}$,

$$
\begin{aligned}
\sigma_{A} & =\frac{\left(\beta_{A} A\right)^{a}}{\left[\left(\beta_{A} A\right)^{a}+\left(\beta_{K} K\right)^{a}+\left(\beta_{H}\left(l_{Y} H\right)\right)^{a}\right]} \\
& =\left(\frac{\left(\beta_{A} A\right)^{a}}{\left[\left(\beta_{A} A\right)^{a}+\left(\beta_{K} K\right)^{a}+\left(\beta_{H}\left(l_{Y} H\right)\right)^{a}\right]^{1 / a}}\right)^{a} \\
& =\left(\frac{\beta_{A} A}{Y}\right)^{a} \\
\sigma_{H} & =\left(\frac{\beta_{H}\left(l_{Y} H\right)}{Y}\right)^{a} \\
\sigma_{K} & =\frac{\left(\beta_{K} K\right)^{a}}{\left[\left(\beta_{A} A\right)^{a}+\left(\beta_{K} K\right)^{a}+\left(\beta_{H}\left(l_{Y} H\right)\right)^{a}\right]} \\
& =\frac{\left(\beta_{A} A\right)^{a}+\left(\beta_{H}\left(l_{Y} H\right)\right)^{a}+\left(\beta_{K} K\right)^{a}-\left(\beta_{A} A\right)^{a}-\left(\beta_{H}\left(l_{Y} H\right)\right)^{a}}{\left[\left(\beta_{A} A\right)^{a}+\left(\beta_{K} K\right)^{a}+\left(\beta_{H}\left(l_{Y} H\right)\right)^{a}\right]} \\
& =1-\left(\sigma_{A}+\sigma_{H}\right)
\end{aligned}
$$

Example 3: Cobb-Douglas Constant Returns to Scale Human Capital Accumulation From (20) and differentiation of (19a) when $\sigma_{A}+\sigma_{H}=1$ gives

$$
g_{Y}=g_{A}=g_{H}
$$

We therefore define $\psi \equiv H / A$. The dynamic evolution of $\psi_{t}$ is subsequently derived from the special case of equations (19a), (19b) and (19c)

$$
g_{\psi} \equiv g_{H}-g_{A}=\left(B_{H} \psi_{t}^{\sigma_{H}-1}-B_{A} \psi_{t}^{\sigma_{H}}\right)
$$


$g_{\psi}=0$ implies a steady state ratio of aggregate human capital to ideas,

$$
\psi=\frac{B_{H}}{B_{A}}
$$

which, by (A2.2), is stable.

Using equations (19a), (19b), (19c) and (A2.3), along a balanced growth path

$$
g_{A}=g_{H}=g_{h}+n=\left(B_{A}\right)^{1-\sigma_{H}}\left(B_{H}\right)^{\sigma_{H}}
$$

The central planner chooses $c, B_{A}$ and $B_{H}$. From (3a)

$$
g_{c}=\frac{1}{\theta}[r-\rho-n]
$$

The condition equivalent to (3b) implies $\lambda=\mu=\gamma$. Substituting for $\lambda=\mu=\gamma$ in (3e) and (3f),

$$
\left(1-\sigma_{H}\right) \frac{Y}{A}=\sigma_{H} \frac{Y}{H}=r
$$

which implies

$$
\frac{B_{H}}{B_{A}}=\frac{H}{A}=\frac{\sigma_{H}}{1-\sigma_{H}}
$$

Derivation of equilibrium with intermediate goods

The decentralised economy

Households Households choose $c$ and $l_{H}$ to maximise (1) subject to the budget constraint

$$
\dot{D}=r D+w\left(1-l_{H}\right) H-c L
$$

where $w$ is wages per unit of employed human capital and $r$ returns per unit of aggregate 
wealth, $D$. The current value Hamiltonian is

$$
\hat{H}=\frac{c^{1-\theta}}{1-\theta}+\lambda\left[r D+w\left(1-l_{H}\right) H-c L\right]+\gamma \psi l_{H} H
$$

where $c, l_{H}$ are control and $D, H$ are state variables. The first order conditions are

$$
\begin{aligned}
c^{-\theta} & =\lambda \\
\lambda w & =\gamma \psi \\
\dot{\bar{\lambda}} & =\rho-r \\
\frac{\lambda}{\dot{\gamma}} & =\rho-\frac{\lambda}{\gamma} w\left(1-l_{H}\right)-\gamma \psi l_{H} \\
\frac{\gamma}{\gamma} &
\end{aligned}
$$

From (A4.1) and (A4.3)

$$
\begin{aligned}
g_{C} & =\frac{1}{\theta}(r-\rho) \\
g_{w} & =r-\psi
\end{aligned}
$$

Firms and markets The market for final goods is perfectly competitive and the price is normalised to 1 . No-arbitrage requires

$$
r=\sigma_{K} \frac{Y}{K}
$$

and equating price and marginal production costs yields

$$
\begin{aligned}
p(i) & =\frac{\sigma_{A} Y}{A x^{\phi}} x(i)^{\phi-1} \\
w & =\sigma_{K} \frac{Y}{l_{H} H}
\end{aligned}
$$


where $p(i)$ is the price of an intermediate good.

Each firm in the intermediate goods sector maximises profits

$$
\pi(i)=(p(i)-1) x(i)
$$

by charging

$$
p(i)=\frac{1}{\phi}
$$

With symmetric demand, $x(i)=x$. From $(\mathrm{A} 4.8)$

$$
A x=\phi \sigma_{A} Y
$$

and (A4.10), profit is

$$
\pi=(1-\phi) \sigma_{A} \frac{Y}{A}
$$

$R \mathscr{G} D$ sector Let $v$ denote the value of innovation. Free entry in $\mathrm{R} \& \mathrm{D}$ requires

$$
w=\delta v
$$

and no-abitrage for investors requires

$$
r=\frac{\pi}{v}+\frac{\dot{v}}{v}
$$

which together imply

$$
\frac{\dot{w}}{w}=r-\frac{\delta \pi}{w}
$$


Equilibrium Substituting from (A4.6), (A4.9) and (A4.12) in (A4.15) gives

$$
l_{Y}^{D}=\frac{1}{1-\phi} \frac{\psi}{\delta} \frac{\sigma_{H}}{\sigma_{A}} \frac{A}{H}
$$

Taking the logarithm and time derivative of (25), after substituting for $x$ from (A4.11) and $g_{Y}=g_{C}=g_{K}$ and $g_{A}=g_{H}$ in steady state,

$$
g_{Y}=\left[\frac{1-\phi}{\phi} \frac{\sigma_{A}}{\sigma_{H}}+1\right] g_{A}
$$

The logarithm and time derivative of (A4.9) and equating with (A4.6) gives (A3.5), where substituting for $g_{l_{Y}}=0, r$ and $g_{Y}$ from (A4.16), (A4.5) and (A4.17), respectively, gives

$$
g_{A}=g_{H}=\frac{(\psi-\rho) \frac{\phi}{1-\phi} \frac{\sigma_{H}}{\sigma_{A}}}{\frac{\phi}{1-\phi} \frac{\sigma_{H}}{\sigma_{A}} \theta+\theta-1}
$$

The planner's problem The current value Hamiltonian is

$$
\hat{H}=\frac{c^{1-\theta}}{1-\theta}+\lambda[Y-c L-A x]+\mu \delta l_{A} H+\gamma \psi l_{H} H
$$

where $c, l_{A}, l_{H}, x$ are control variables and $K, A, H$ are state variables. From (29)

$$
\lambda\left[\sigma_{A} \frac{Y}{x}-A\right]=0
$$

From (28)

$$
\rho-\frac{\dot{\mu}}{\mu}=\frac{\lambda}{\mu}\left(\frac{Y}{x}-A\right)
$$

From the second equality in (3b), $\mu \delta=\gamma \psi$. Differentiating with respect to time, we can substitute for $\dot{\mu}$ and $\mu$ in (3e). Equating with (3f) and further substituting for $\mu \delta$ and $\gamma \psi$ 
in $(3 \mathrm{f})$

$$
l_{Y}^{C P}=\frac{\phi}{1-\phi} \frac{\psi}{\delta} \frac{\sigma_{H}}{\sigma_{A}} \frac{A}{H}
$$

Taking the logarithm and time derivative of (25), substituting for $x$ from (A3.1) and $g_{Y}=g_{C}=g_{K}$ and $g_{A}=g_{H}$ in steady state, gives the same equilibrium $g_{Y}$ as (A4.17). From (3b), (3d) and (3f)

$$
g_{Y}-g_{l_{Y}}-g_{H}=r-\psi
$$

where substituting for $g_{l_{Y}}=0, r$ and $g_{Y}$ from (A3.3), (A1.3) and (A4.17), respectively, gives the same equilibrium $g_{A}$ as (A4.18). 